ANALYSIS \& PDE Volume $9 \quad$ No. $3 \quad 2016$

PABlo ANGULO-ARDOY, DANIEL FARACO, 1. LUIS GUIJARRO AND ALBERTO RUIZ

OBSTRUCTIONS TO THE EXISTENCE OF LIMITING CARLEMAN WEIGHTS 


\title{
OBSTRUCTIONS TO THE EXISTENCE OF LIMITING CARLEMAN WEIGHTS
}

\author{
Pablo Angulo-Ardoy, Daniel Faraco, Luis Guijarro and Alberto Ruiz
}

\begin{abstract}
We give a necessary condition for a Riemannian manifold to admit limiting Carleman weights in terms of its Weyl tensor (in dimensions 4 and higher), or its Cotton-York tensor in dimension 3. As an application, we provide explicit examples of manifolds without limiting Carleman weights and show that the set of such metrics on a given manifold contains an open and dense set.
\end{abstract}

\section{Introduction}

The inverse problem posed by Calderón asks for the determination of the conductivity of a medium by making voltage-to-current measurements in the boundary. The problem in the current form started with the seminal work of Calderón [1980] and research on it has been very intense. An outstanding problem is the case of anisotropic conductivities. At least in dimension $n \geqslant 3$, the right formalism seems to be the language of differential geometry. Namely for $(M, g)$, a Riemannian manifold with boundary, and $\triangle_{g}$, the corresponding Laplace-Beltrami operator, does the Dirichlet-to-Neumann map determine the metric $g$ up to a gauge transformation? The problem seemed out of reach, apart from the real analytic class (see [Kohn and Vogelius 1984; 1985]). However, a recent breakthrough in [Dos Santos Ferreira et al. 2009] allows one to solve several inverse problems in the Riemannian setting for a larger class of Riemannian manifolds. We refer to [Dos Santos Ferreira et al. 2009; 2013b; Salo 2013] for detailed accounts of these results, and recall the following theorem as an illustration. For reconstruction, see [Kenig et al. 2011] and for stability, see [Caro and Salo 2014].

Theorem 1.1 [Dos Santos Ferreira et al. 2009, Theorem 1.7; 2013a, Theorem 1.1]. Let (M, g) be an admissible Riemannian manifold of dimension $n \geqslant 3$ with boundary and $q_{1}, q_{2}$ be two potentials in $L^{n / 2}(M)$. Assume that 0 is not a Dirichlet eigenvalue for the corresponding Schrödinger operator $\mathcal{L}_{q_{i}}=-\triangle_{g}+q_{i}$. If $\Lambda_{q_{1}}=\Lambda_{q_{2}}$, then $q_{1}=q_{2}$.

A precise definition of admissibility is given in [Dos Santos Ferreira et al. 2009, Definition 1.5], but a necessary condition in that paper for a manifold $(M, g)$ to be so was the existence of a so-called limiting Carleman weight (LCW for short). It turns out that this is a conformally invariant notion, as the following theorem shows:

The authors were supported by research grants MTM2011-22612, MTM2011-28198, MTM2014-57769-1-P and MTM201457769-3-P from the Ministerio de Ciencia e Innovación (MCINN), by MINECO: ICMAT Severo Ochoa project SEV-2011-0087, and by the ERC 301179 .

MSC2010: primary 35R30, 53A30; secondary 58J32.

Keywords: Calderón problem, limiting Carleman weights, conformal geometry, Cotton-York tensor, Weyl tensor, conformal to a product. 
Theorem 1.2 [Dos Santos Ferreira et al. 2009, Theorem 1.2]. If $(M, g)$ is an open manifold having a limiting Carleman weight, then some conformal multiple of the metric $g$, called $\tilde{g} \in[\mathrm{g}]$, admits a parallel unit vector field. For simply connected manifolds, the converse is true.

Recall that a vector field $X$ is parallel if $\nabla X=0$ and that in a simply connected manifold, $X$ is parallel if and only if it is a Killing field (e.g., $\mathcal{L}_{X} g=0$ ) and also a gradient field. It was proven in [Dos Santos Ferreira et al. 2009] that if $\tilde{g}$ admits a parallel vector field $X$, there exist local coordinates such that $X=\partial_{1}$ and

$$
\tilde{g}\left(x_{1}, x^{\prime}\right)=\left(\begin{array}{cc}
1 & 0 \\
0 & g_{0}\left(x^{\prime}\right)
\end{array}\right) \text { and hence } g(x)=e^{2 f(x)}\left(\begin{array}{cc}
1 & 0 \\
0 & g_{0}\left(x^{\prime}\right)
\end{array}\right) .
$$

In other words, around each point, $\tilde{g}=e \oplus g_{0}$, where $g_{0}$ is the metric of an $(n-1)$-manifold and $e$ is the euclidean metric in $\mathbb{R}$.

Here we concentrate on the local existence of limiting Carleman weights for a given metric $g$. Thus we can consider the manifolds as being simply connected, and the existence of limiting Carleman weights is therefore equivalent to having parallel vector fields after a conformal change of the metric. This characterization is very elegant but it has the drawback that it requires information about the whole conformal class of $g$. It would be desirable to have a criterion that depends on the metric $g$ itself in an invariant manner. It seems natural to look at this question in terms of the Weyl curvature tensor, which as a $(1,3)$-tensor is a conformal invariant. In dimension $n \geqslant 4$, being conformally flat is equivalent to the Weyl tensor being zero.

For the case of parallel vector fields, we prove:

Theorem 1.3. Let $(M, g)$ be a Riemannian manifold of dimension $n \geqslant 4$. Assume that a metric $\tilde{g} \in[g]$ admits a parallel vector field. Then for any $p \in M$, there is a tangent vector $v \in T_{p} M$ such that the Weyl tensor of any metric in $[g]$ satisfies $W_{p}\left(v \wedge v^{\perp}\right) \subset v \wedge v^{\perp}$. In particular, for any $p \in M$, we have $W_{p} \in S^{2}\left(\Lambda^{2}\left(T_{p}^{*} M\right)\right)$ has at least $n-1$ linearly independent eigenvectors that are simple.

Recall that an element of $\Lambda_{p}^{2}(M)$ is simple if it is equal to $v \wedge w$ for $v, w \in T_{p} M$. In the above theorem, we are considering $W_{p}$ as a curvature operator as defined, for instance, in [Besse 1987] and given a vector $v \in T_{p} M$, we define $v^{\perp} \in T_{p} M$ to be its orthogonal complement, that is, $v \oplus v^{\perp}=T_{p} M$. An algebraic Weyl operator (Weyl tensor) in a euclidean vector space $V$ is a symmetric operator on the space $\Lambda^{2} V$ that satisfies the Bianchi and the Ricci conditions (see Section 2, equations (3) and (4) for the definitions). To facilitate the reading, we include a brief overview of curvature operators in Section 2. We also give a special name to algebraic Weyl operators satisfying the condition in the above theorem.

Definition 1.4. Let $W$ be a Weyl tensor. We say that $W$ satisfies the eigenflag condition if and only if there is a vector $v \in V$ such that $W\left(v \wedge v^{\perp}\right) \subset v \wedge v^{\perp}$.

The following is an easy corollary of Theorem 1.3.

Corollary 1.5. Let $(M, g)$ be a 4-dimensional Riemannian manifold such that some $\tilde{g} \in[g]$ admits a parallel vector field. Then all the eigenvectors of the Weyl operator of $g$ are simple.

The theorem gives a simple algebraic condition to decide whether a given Riemannian manifold can admit a parallel vector field after a conformal change. Hence our theorem yields a quick way to decide that a given metric does not admit limiting Carleman weights; we illustrate this in Section 4 by showing 
that any manifold locally isometric to $\mathbb{C P}^{2}$ with its Fubini-Study metric does not fall into this class. However, the metric is analytic so Calderón's problem can be solved by unique continuation from the boundary, at least for analytic potentials.

Notice that conformal geometry in dimensions $n=2$ and $n=3$ is characterized differently. In dimension $n=2$, every manifold is conformally flat due to the existence of isothermal coordinates. Dimension $n=3$ is also special as conformal flatness is characterized by the vanishing of the Cotton tensor. Notice that in the presence of conformal flatness, direct proofs are available as long as the conformal parametrization is invertible. In analogy with higher dimensions, the existence of conformally parallel vector fields (and thus the existence of limiting Carleman weights) can be read algebraically from the Cotton-York tensor.

Theorem 1.6. Let $n=3$. If a metric $\tilde{g} \in[g]$ admits a parallel vector field then, for any $p \in M$, there is a tangent vector $v \in T_{p} M$ such that

$$
C Y_{p}(v, v)=C Y_{p}\left(w_{1}, w_{2}\right)=0
$$

for any pair of vectors $w_{1}, w_{2} \in v^{\perp}$.

In the above theorem, the Cotton-York tensor $C Y$ is understood as a $(0,2)$-tensor. The characterization can be read easily from the matrix representation of the Cotton-York tensor in any basis.

Corollary 1.7. The above condition is equivalent to $\operatorname{det}\left(C Y_{p}\right)=0$.

Finally, we end our study of the 3-dimensional case using Theorem 1.6 and Corollary 1.7 to determine which of the eight Thurston geometries admit limiting Carleman weights. The motivation for such a question spurs from the geometrization theorem, since any closed oriented 3-dimensional manifold arises as union of pieces admitting one of these eight geometries.

Theorem 1.8. Among the eight Thurston geometries, only the Nil and $\widetilde{\mathrm{SL}_{2}(\mathbb{R})}$-geometries do not admit limiting Carleman weights. The others are admissible in the sense of [Dos Santos Ferreira et al. 2009].

In the last section, we show that the set of metrics not admitting LCWs contains an open and dense subset of the space of all the metrics. A precise statement is contained in the next result:

Theorem 1.9. Let $U$ be an open submanifold of some compact manifold $M$ without boundary having dimension $n \geqslant 3$. The set of Riemannian metrics on $M$ for which no limiting Carleman weight exists on $U$ contains an open and dense subset of the set of all metrics, endowed with the $C^{3}$-topology for $n=3$, and the $C^{2}$-topology for $n \geqslant 4$.

Remark 1.10. If a Riemannian metric on $U$ admits an LCW, then Theorem 1.3 shows that its Weyl tensor satisfies the eigenflag condition at every point of $U$. We make use of that fact in our proof of Theorem 1.9, fixing a point $p_{0}$, and proving that the set of metrics whose Weyl tensor at $p_{0}$ does not satisfy the eigenflag condition is open and dense.

The proof of Theorem 1.9 gives indeed a constructive method for building explicit metrics that do not admit an LCW near any given Riemannian metric by adding a "bump" at a certain point. In Section 4 and the subsection beginning on page 584, we show explicit examples of classical homogeneous manifolds that do not admit local LCWs at any point of $U$. 
In the companion paper [Angulo-Ardoy 2015], it is shown that the set of Riemannian metrics on $U$ that do not admit a locally defined LCW at any point is also open and dense. This generalizes [Liimatainen and Salo 2012, Corollary 1.3], where it is proven that this set is residual.

\section{Tensors in conformal geometry}

The proof relies on the decomposition of the curvature tensor and its behaviour under conformal transformations. We denote by $R, S$ and Ric the $(0,4)$-curvature, Schouten and Ricci tensors respectively, and by $s$ the scalar curvature. Recall

$$
\begin{aligned}
& S=\frac{1}{n-2}\left(\operatorname{Ric}-\frac{1}{2(n-1)} s g\right), \\
& R=W+S \otimes g,
\end{aligned}
$$

where $\otimes$ is the Kulkarni-Nomizu product of two symmetric 2-tensors, which is defined by

$$
(\alpha \oplus \beta)_{i j k l}=\alpha_{i k} \beta_{j l}+\beta_{i k} \alpha_{j l}-\alpha_{i l} \beta_{j k}-\alpha_{j k} \beta_{i l},
$$

and $R$ and $W$ are understood as $(0,4)$-tensors.

In the proof of Theorem 1.3, we consider $W$ as an algebraic curvature operator; for a fuller treatment of such objects, we refer the reader to [Besse 1987], but for completeness we include here a short description. Consider the curvature at a point $p$ as a $(0,4)$-tensor; its symmetries allow us to consider it as a symmetric linear endomorphism $\rho_{p}$ of the space of bivectors $\Lambda^{2}\left(T_{p}^{*} M\right)$, that is, $\rho_{p} \in S^{2}\left(\Lambda^{2}\left(T_{p}^{*} M\right)\right)$. Now the first Bianchi identity induces a projector onto the 4 -forms, considered as symmetric endomorphisms of the space of bivectors:

$$
b(R)(x, y, z, t)=\frac{1}{3}(R(x, y, z, t)+R(y, z, x, t)+R(z, x, y, t)),
$$

so that $S^{2}\left(\Lambda^{2}\left(T_{p}^{*} M\right)\right)=\operatorname{ker}(b) \oplus \operatorname{Im}(b)$, where the elements of $\operatorname{ker}(b)$ are called the algebraic curvature operators. It turns out the Weyl tensors are curvature operators in the kernel of the Ricci contraction. That is, if we define $r: S^{2}\left(\Lambda^{2}\left(T_{p}^{*} M\right)\right) \rightarrow S^{2}\left(T_{p}^{*}(M)\right)$ by

then

$$
r(R)(x, y)=\operatorname{Tr}[R(x, \cdot, y, \cdot)]
$$

$$
\mathcal{W}\left(T_{p} M\right)=\operatorname{ker}(b) \cap \operatorname{ker}(r) .
$$

We would like to remark on one property of the space of Weyl tensors. Any rotation $\rho \in \operatorname{SO}(V)$ induces a rotation $B(\rho)$ on the space of bivectors, where $B(\rho)(v \wedge w)=\rho(v) \wedge \rho(w)$. The space of Weyl tensors is invariant under all such rotations (see [Besse 1987, 1.114]):

$$
W_{p} \in \mathcal{W}\left(T_{p} M\right) \quad \Longleftrightarrow \quad B(\rho) \circ W_{p} \circ B(\rho)^{t} \in \mathcal{W}\left(T_{p} M\right) .
$$

In our formulation of Theorem 1.3, we used the isomorphism induced by $g$ between $\Lambda^{2}\left(T_{p}^{*} M\right)$ and $\Lambda^{2}\left(T_{p} M\right)$ to consider $W_{p}$ as a symmetric endomorphism of the latter space. Thus, given a simple bivector $x \wedge y \in \Lambda^{2}\left(T_{p} M\right)$, we have that $W_{p}(x \wedge y)$ is the only bivector (not necessarily simple) such that

$$
\left\langle W_{p}(x \wedge y), z \wedge t\right\rangle=\left\langle W_{p}(x, y) z, t\right\rangle
$$

for any $z, t \in T_{p} M$, where the $W_{p}$ in the right-hand side is considered as a (1,3)-tensor. 
When dealing with a 4-dimensional manifold $M$, we make use of the Hodge operator (or, more precisely, of its equivalent in bivectors). This is a linear map $*: \Lambda_{p}^{2} M \rightarrow \Lambda_{p}^{2} M$ defined as

$$
\langle * \omega, \tau\rangle=\left\langle\omega \wedge \tau, e_{1} \wedge e_{2} \wedge e_{3} \wedge e_{4}\right\rangle
$$

for an oriented orthonormal basis $\left\{e_{i}\right\}$ of $T_{p} M$. Since $*$ is self-adjoint and $(*)^{2} \omega=\omega$ for any bivector, there is a splitting

$$
\Lambda_{p}^{2}=\Lambda^{+} \oplus \Lambda^{-}
$$

into eigenspaces with eigenvalues 1 and -1 respectively. Each eigenspace has dimension $3: \Lambda^{+}$is spanned by the bivectors $e_{1} \wedge e_{2}+e_{3} \wedge e_{4}, e_{1} \wedge e_{3}+e_{4} \wedge e_{2}$ and $e_{1} \wedge e_{4}+e_{2} \wedge e_{3}$ and $\Lambda^{-}$by the bivectors $e_{1} \wedge e_{2}-e_{3} \wedge e_{4}, e_{1} \wedge e_{3}-e_{4} \wedge e_{2}$ and $e_{1} \wedge e_{4}-e_{2} \wedge e_{3}$.

This gives a corresponding splitting for algebraic curvature operators $R$ :

$$
R=\left(\begin{array}{cc}
\frac{s}{12} \mathrm{Id}+W^{+} & Z \\
Z^{t} & \frac{s}{12} \mathrm{Id}+W^{-}
\end{array}\right),
$$

where $W=W^{+} \oplus W^{-}$and $Z=\left(\right.$ Ric $\left.-\frac{s}{4} g\right) \oplus g$ (see [Besse 1987, 1.126-1.128]).

Another important tensor in conformal geometry is the Cotton tensor. It is a $(0,3)$-tensor defined as

$$
C_{i j k}=\left(\nabla_{i} S\right)_{j k}-\left(\nabla_{j} S\right)_{i k},
$$

where the notation $\left(\nabla_{a} S\right)_{b c}$ stands for $\left(\nabla_{\partial_{a}} S\right)\left(\partial_{b}, \partial_{c}\right)$, so that

$$
\left(\nabla_{a} S\right)_{b c}=\partial_{a}\left(S\left(\partial_{b}, \partial_{c}\right)\right)-S\left(\nabla_{a} \partial_{b}, \partial_{c}\right)-S\left(\partial_{b}, \nabla_{a} \partial_{c}\right) .
$$

The Cotton tensor has the symmetries

$$
\begin{aligned}
C_{i j k} & =-C_{j i k}, \\
C_{i j k}+C_{j k i}+C_{k i j} & =0, \\
g^{i j} C_{i j k} & =0, \\
g^{i k} C_{i j k} & =0 .
\end{aligned}
$$

The first three are straightforward, and the last follows from the second Bianchi identity (see [York 1971]).

If the metric is changed within its conformal class to $\tilde{g}=e^{2 f} g$, the $(1,3)$-Weyl tensor is unchanged, the $(0,4)$-Weyl tensor changes as $\widetilde{W}=e^{2 f} W$, and the Cotton tensor changes as

$$
\widetilde{C}(x, y, z)=C(x, y, z)-W(x, y, z, \nabla f) .
$$

Indeed, conformal flatness is characterized, at any dimension $n \geqslant 3$, by the vanishing of both the Cotton and Weyl tensors at all points (see, for example, [Hertrich-Jeromin 2003, p. 5] for the classical proof and [Liimatainen and Salo 2015] for less regular metrics).

For $n \geqslant 4$, the Cotton tensor is the divergence of the Weyl tensor:

Proposition 2.1. If $n \geqslant 3$, then $\left(\nabla_{l} W\right)_{i j k}^{l}=(n-3) C_{i j k}$.

Thus the Cotton tensor vanishes if the Weyl tensor vanishes.

In dimension $n=3$, the Weyl tensor always vanishes, and conformal flatness has to be read directly from the Cotton tensor. This is conformally invariant, and it is equivalent to the so-called Cotton-York 
tensor. This new tensor is defined by considering the Cotton tensor as a map $C_{p}: T_{p} M \rightarrow \Lambda^{2}\left(T_{p}^{*} M\right)$ (thanks to the antisymmetry of $C$ with respect to its first two entries) and composing with the Hodge star operator $*: \Lambda^{2}\left(T_{p}^{*} M\right) \rightarrow T_{p}^{*} M$. This gives a $(0,2)$-tensor that turns out to be symmetric and trace-free, but not conformally invariant. The Cotton-York tensor also appears in the literature as a $(1,1)$-tensor after raising one index.

In a patch with coordinates $x^{1}, x^{2}, x^{3}$, the Hodge star has the expression

$$
*\left(d x^{i} \wedge d x^{j}\right)=\sum g_{l k} \frac{\epsilon^{i j l}}{\sqrt{\operatorname{det}(g)}} d x^{k},
$$

where $\epsilon^{i j l}$ is the signature of the permutation $(i, j, l)$ (it takes the values 0,1 and -1 ). So from

$$
C=\sum C_{i j k} d x^{i} \otimes d x^{j} \otimes d x^{k}=\frac{1}{2} \sum C_{i j k}\left(d x^{i} \wedge d x^{j}\right) \otimes d x^{k},
$$

the following expression for the $(0,2)$-version of the Cotton-York tensor follows:

$$
C Y_{i j}=\frac{1}{2} C_{k l i} g_{j m} \frac{\epsilon^{k l m}}{\sqrt{\operatorname{det} g}}=g_{j m}\left(\nabla_{k} S\right)_{l i} \frac{\epsilon^{k l m}}{\sqrt{\operatorname{det} g}} .
$$

It follows from (8) that this tensor is symmetric and its trace is zero:

$$
\begin{aligned}
C Y_{i j} & =C Y_{j i}, \\
g^{i j} C Y_{i j} & =C Y_{i}^{i}=0 .
\end{aligned}
$$

Remark 2.2. The reader may notice, looking at (9), that the Cotton-York tensor is not conformally invariant. However, if the metric $g$ is replaced by $\lambda g$, the Cotton-York tensor is scaled by $\lambda^{-1 / 2}$ so, in particular, the determinant of the tensor is zero if and only if it is zero for any conformal metric. The $(1,1)$-version of the Cotton-York tensor is not conformally invariant either. We remark that our computation of the scaling factor differs from the one found in the literature [York 1971].

\section{Proof of Theorem 1.3}

The $(1,3)$-Weyl tensor is invariant under conformal changes of the metric. Thus, thanks to Theorem 1.2, we can assume that $g$ admits a parallel vector field $X$. As in [Dos Santos Ferreira et al. 2009], we notice that in the appropriate semigeodesic coordinates, $X=e_{1}$ and the metric is written as

$$
\tilde{g}\left(x_{1}, x^{\prime}\right)=\left(\begin{array}{cc}
1 & 0 \\
0 & g_{0}\left(x^{\prime}\right)
\end{array}\right) .
$$

For any set of coordinates, $e_{1}$ is parallel if and only if $R_{1 i j k}=0$ (the sufficiency follows from Frobenius' theorem). Moreover, notice that $g_{1 j}=0$ for all $j \geqslant 2$. Thus, by the formula of the Schouten tensor, it holds that in these coordinates, $S_{1 j}=0$ for all $j \geqslant 2$. Now for $j, k, l \geqslant 2$,

$$
(S \oplus g)_{1 j k l}=S_{1 k} g_{j l}+S_{j l} g_{1 k}-S_{1 l} g_{j k}-S_{j k} g_{1 l}=0,
$$

and by the decomposition of the curvature tensor,

$$
W_{1 j k l}=R_{1 j k l}-(S \otimes g)_{1 j k l}=0 .
$$


Recall that $W$ acts on bivectors by

$$
W\left(e_{i} \wedge e_{j}\right)=\sum_{k, l} W_{i j k l} e_{k} \wedge e_{l} .
$$

Given $p \in M$, let $v=X_{p}=e_{1}$; thus $g_{1 j}=\delta_{1 j}$; in these coordinates, $e_{1} \wedge e_{1}^{\perp}$ is invariant. In other words, for every $j, k, l \neq 1$,

$$
\left\langle W\left(e_{1} \wedge e_{j}\right), e_{k} \wedge e_{l}\right\rangle=0=W_{1 j k l} .
$$

Therefore $W\left(v \wedge v^{\perp}\right) \subset v \wedge v^{\perp}$, and the first part of Theorem 1.3 is proved. Finally, $v \wedge v^{\perp}$ is an $(n-1)$-dimensional subspace of simple bivectors; thus it contains $n-1$ linearly independent simple eigenbivectors of $W$.

Proof of Corollary 1.5. Let $v \in T_{p} M$ be the vector given by Theorem 1.3. Since $\Lambda^{2}\left(v^{\perp}\right)$ is orthogonal to $v \wedge v^{\perp}$, and $v \wedge v^{\perp}$ is invariant by $W$, we know that $W$ also leaves $\Lambda^{2}\left(v^{\perp}\right)$ invariant. But $v^{\perp}$ being 3-dimensional implies that every element of $\Lambda^{2}\left(v^{\perp}\right)$ is simple, finishing the proof.

\section{Examples of manifolds without LCWs}

This section provides explicit examples of Riemannian manifolds without any LCWs. Namely, this:

Theorem 4.1. Let $\mathbb{C P}^{2}$ be the complex projective space with its Fubini-Study metric $g_{\text {can. Then any }}$ subdomain $\Omega \subset \mathbb{C P}^{2}$ with boundary does not admit an LCW.

Proof. Since $\mathbb{C P}^{2}$ is 4-dimensional, we will make use of the decomposition

$$
\Lambda_{p}^{2} \mathbb{C} \mathbb{P}^{2}=\Lambda^{+} \oplus \Lambda^{-}
$$

induced by the Hodge operator $*: \Lambda_{p}^{2} \mathbb{C P}^{2} \rightarrow \Lambda_{p}^{2} \mathbb{C P}^{2}$ as was explained in Section 2 .

Use $J: T_{p} \mathbb{C P}^{2} \rightarrow T_{p} \mathbb{C P ^ { 2 }}$ to denote the canonical complex structure of $\mathbb{C P}^{2}$ and let $\left\{e_{i}\right\}$ be an orthonormal basis of $T_{p} \mathbb{C} \mathbb{P}^{2}$, with $e_{2}=J e_{1}, e_{4}=J e_{3}$. A basis of $\Lambda_{p}^{2} \mathbb{C} \mathbb{P}^{2}$ is given by

$$
\phi_{1}=e_{1} \wedge e_{2}+e_{3} \wedge e_{4}, \quad \phi_{2}=e_{1} \wedge e_{3}-e_{2} \wedge e_{4}, \quad \phi_{3}=e_{1} \wedge e_{4}+e_{2} \wedge e_{3}
$$

for its self-dual component, and

$$
\psi_{1}=e_{1} \wedge e_{2}-e_{3} \wedge e_{4}, \quad \psi_{2}=e_{1} \wedge e_{3}+e_{2} \wedge e_{4}, \quad \psi_{3}=e_{1} \wedge e_{4}-e_{2} \wedge e_{3}
$$

for its anti-self-dual part.

The curvature of $\mathbb{C P}^{2}$ is computed in several texts in Riemannian geometry; we give a quick overview here, but see [do Carmo 1992, p. 189] for more details. Viewing $S^{5}$ as the unit sphere in $\mathbb{C}^{3}$, and $\mathbb{C} \mathbb{P}^{2}$ as the basis of a Riemannian submersion under the action of $S^{1}$ on $S^{5}$ given by $z \cdot\left(z_{1}, z_{2}, z_{3}\right)=\left(z z_{1}, z z_{2}, z z_{3}\right)$, the sectional curvature of a 2 -plane in $\mathbb{C P}^{2}$ is

$$
K(X, Y)=1+3 \cos ^{2} \phi,
$$

where $X, Y$ is an orthonormal basis of the plane in $\mathbb{C P}^{2}$, and $\cos \phi$ is the hermitian product $\langle\bar{X}, i \bar{Y}\rangle$ of the horizontal lifts $\bar{X}, \bar{Y}$ of $X, Y$ respectively to $S^{5}$. From here it is easy to see that the sectional curvatures of $\mathbb{C P} \mathbb{P}^{2}$ take values between 1 and 4. Since norms of horizontal lifts agree with those of the vectors in 
the base, $0 \leqslant\langle\bar{X}, i \bar{Y}\rangle \leqslant 1$. Therefore $K(X, Y)=1$ only when $\langle\bar{X}, i \bar{Y}\rangle=0$; since the complex structure of $\mathbb{C P} \mathbb{P}^{2}$ is induced by that of $\mathbb{C}^{3}$, this happens only when the plane $\sigma=\{X, Y\}$ satisfies $J \sigma \perp \sigma$. On the other hand, a 2-plane $\sigma$ will have $K(\sigma)=4$ if and only if $\sigma$ is complex, i.e, $J \sigma=\sigma$.

To recover the full curvature operator from the sectional curvature, either use an explicit formula for the terms of the curvature in terms of the sectional curvatures, as the one in [Cheeger and Ebin 1975, p. 16], or continue using O'Neill's formula for the curvature terms $\langle R(x, y) z, w\rangle$ in $\mathbb{C P}^{2}$ in terms of the corresponding curvature terms in $S^{5}$ and O'Neill's $A$-tensor, as in [do Carmo 1992, p. 187, Exercise 10(a)]. The reader will also find [Sakai 1996, pp. 76-77] useful, which, in spite of defining the curvature tensor differently, makes explicit the relation between the complex structure of $\mathbb{C P ^ { 2 }}$ and the submersion $S^{5} \rightarrow \mathbb{C P}^{2}$.

The only nonvanishing components of the curvature tensor are then

$$
\begin{aligned}
\left\langle R\left(e_{1}, e_{2}\right) e_{1}, e_{2}\right\rangle & =\left\langle R\left(e_{3}, e_{4}\right) e_{3}, e_{4}\right\rangle=4, \\
\left\langle R\left(e_{1}, e_{3}\right) e_{1}, e_{3}\right\rangle=\left\langle R\left(e_{1}, e_{4}\right) e_{1}, e_{4}\right\rangle & =\left\langle R\left(e_{2}, e_{3}\right) e_{2}, e_{3}\right\rangle=\left\langle R\left(e_{2}, e_{4}\right) e_{2}, e_{4}\right\rangle=1
\end{aligned}
$$

for the sectional curvatures and

$$
\left\langle R\left(e_{1}, e_{2}\right) e_{3}, e_{4}\right\rangle=2, \quad\left\langle R\left(e_{1}, e_{3}\right) e_{2}, e_{4}\right\rangle=1, \quad\left\langle R\left(e_{1}, e_{4}\right) e_{2}, e_{3}\right\rangle=-1
$$

for the mixed terms.

In the space of bivectors and with the $\phi_{i}, \psi_{i}$ as above, the curvature operator $R_{p}$ satisfies

$$
\begin{array}{lll}
R_{p}\left(\phi_{1}\right)=6 \phi_{1}, & R_{p}\left(\phi_{2}\right)=0, & R_{p}\left(\phi_{3}\right)=0, \\
R_{p}\left(\psi_{1}\right)=2 \psi_{1}, & R_{p}\left(\psi_{2}\right)=2 \psi_{2}, & R_{p}\left(\psi_{3}\right)=2 \psi_{3} .
\end{array}
$$

Thus the curvature operator $R_{p}$ of $g_{\text {can }}$ is written as

$$
R_{p}=\left(\begin{array}{cc}
6 E & 0 \\
0 & 2 I
\end{array}\right),
$$

where $I$ is the $3 \times 3$ identity matrix, and $E$ is the matrix

$$
E=\left(\begin{array}{lll}
1 & 0 & 0 \\
0 & 0 & 0 \\
0 & 0 & 0
\end{array}\right) \text {. }
$$

A simple computation, using (6), yields

$$
W_{p}^{+}=\left(\begin{array}{rrr}
4 & 0 & 0 \\
0 & -2 & 0 \\
0 & 0 & -2
\end{array}\right), \quad W_{p}^{-}=0 .
$$

Observe that every eigenvector of $W_{p}$ belongs to either $\Lambda^{+}$or $\Lambda^{-}$, which contain no simple eigenvectors. Hence no eigenvector of $W_{p}$ is simple, which, by Corollary 1.5 , implies that no subdomain of $\left(\mathbb{C P}^{2}, g_{f s}\right)$ admits an LCW.

Similar arguments can be used in higher dimensions to rule out domains in $\mathbb{C P}^{n}$ or other suitable symmetric spaces. 


\section{The 3-dimensional case}

\section{Restrictions on the Cotton-York tensor.}

Proof of Theorem 1.6. Since Theorem 1.6 is formulated at some fixed point $p \in M$, we can assume that everything is local. Recall that in semigeodesic coordinates, the metric is independent of $x_{1}$, and that

$$
g_{1 j}=0=S_{1 j}=S_{j 1}=0
$$

if $j \neq 1$. It follows also that

$$
0=\Gamma_{1 j}^{k}=\Gamma_{j 1}^{k}=\Gamma_{j k}^{1} .
$$

These identities simplify the expression of the Cotton-York tensor: if either $i, l$ or $k$ is equal to 1 , then

$$
\left(\nabla_{k} S\right)_{l i}=\partial_{k}\left(S_{l i}\right) .
$$

Now for $i \neq 1 \neq j$, we notice that $m \neq 1$ for each nonzero term in the sum:

$$
C Y_{i j}=g_{j m}\left(\nabla_{k} S\right)_{l i} \frac{\epsilon^{k l m}}{\sqrt{\operatorname{det} g}} .
$$

Thus for $\epsilon^{k l m} \neq 0$, necessarily $k$ or $l$ are equal to 1 , and hence

$$
C Y_{i j}=g_{j m} \partial_{k} S_{l i} \frac{\epsilon^{k l m}}{\sqrt{\operatorname{det} g}}=0
$$

using that $\partial_{1} S_{l i}=0=S_{1 i}$ for $i \neq 1$.

Similarly,

$$
\sqrt{\operatorname{det} g} C_{11}=g_{1 m} \partial_{k} S_{l 1} \epsilon^{k l m}=\partial_{k} S_{l 1} \epsilon^{k l 1}=0 .
$$

These equations yield that $v=\partial / \partial_{x_{1}}$ is the vector required in Theorem 1.6.

In fact, since the Cotton tensor is invariant after conformal changes of the metric, we can assume that $M$ is isometric to $\mathbb{R} \times \Sigma$, where $\Sigma$ is a surface. Taking coordinates $\left(x_{1}, x_{2}, x_{3}\right)$, with $t=x_{1}$ and $\left(x_{2}, x_{3}\right)$ isothermal coordinates of $\Sigma$, the metric reads as $g=d x_{1}^{2}+e^{f}\left(d x_{2}^{2}+d x_{3}^{2}\right)$ for some function $f\left(x_{2}, x_{3}\right)$ on $\Sigma$. In these coordinates, a simple expression of the full Cotton-York tensor is available. Namely, the Ricci tensor takes the values

the scalar curvature is

$$
\operatorname{Ric}_{1 i}=0, \quad \operatorname{Ric}_{22}=\operatorname{Ric}_{33}=-\frac{1}{2}(\Delta f), \quad \operatorname{Ric}_{23}=0,
$$

$$
s=-(\Delta f) e^{-f},
$$

the Schouten tensor equals

$$
\operatorname{Ric}_{11}=\frac{1}{4}(\Delta f) e^{-f}, \quad \operatorname{Ric}_{22}=\operatorname{Ric}_{33}=-\frac{1}{4}(\Delta f), \quad \operatorname{Ric}_{12}=\operatorname{Ric}_{13}=\operatorname{Ric}_{23}=0,
$$

and a further calculation using formula (9) yields the following explicit formula for the Cotton-York tensor:

$$
\begin{aligned}
& C Y_{12}=C Y_{21}=-\frac{1}{4}\left(\Delta f \partial_{3} f-\partial_{3}(\Delta f)\right) e^{-f}, \\
& C Y_{13}=C Y_{31}=\frac{1}{4}\left(\Delta f \partial_{2} f-\partial_{2}(\Delta f)\right) e^{-f} .
\end{aligned}
$$


The Cotton-York tensor of the product of $\mathbb{R}$ with a surface $\Sigma$ in isothermal coordinates can also be expressed as

$$
C Y=\frac{1}{2} d x_{1} \cdot(* d s)
$$

where $\cdot$ is the symmetric product of forms, $s$ is the scalar curvature of the surface, and $*$ is the Hodge star operator of the surface, which sends the 1-form $d s$ to an orthogonal 1-form on $\Sigma$.

Proof of Corollary 1.7. Corollary 1.7 follows from this lemma:

Lemma 5.1. Let $V$ be a 3-dimensional euclidean space, and $A: V \rightarrow V$ be a symmetric endomorphism. Then there exists a 2-dimensional subspace $P$ such that for any $v_{1}, v_{2} \in P, w \in P^{\perp}$, we have

$$
\left\langle A v_{1}, v_{2}\right\rangle=\langle A w, w\rangle=0
$$

if and only if $\operatorname{det}(A)=\operatorname{Tr}(A)=0$.

Proof. The "only if" part is clear: Let $e_{1}, e_{2} \in P$ and $e_{3} \in P^{\perp}$ form an orthonormal basis. The expression of $A$ in these coordinates is

$$
A=\left(\begin{array}{lll}
0 & 0 & a \\
0 & 0 & b \\
a & b & 0
\end{array}\right)
$$

Thus the conditions on the determinant and the trace of $A$ are obvious.

For the converse, first notice that since it is symmetric, we can diagonalize $A$. Our conditions imply the existence of $\lambda_{1} \in \mathbb{R}$ and an orthonormal basis $v_{1}, v_{2}, v_{3}$ such that

$$
A=\left(\begin{array}{ccc}
\lambda_{1} & 0 & 0 \\
0 & -\lambda_{1} & 0 \\
0 & 0 & 0
\end{array}\right) \text {. }
$$

The desired plane $P$ is the span of $\left\{v_{1}+v_{2}, v_{3}\right\}$. Namely for $t_{1}, t_{2} \in \mathbb{R}$,

$$
\left\langle A\left(t_{1}\left(v_{1}+v_{2}\right)+t_{2} v_{3}\right), t_{1}\left(v_{1}+v_{2}\right)+t_{2} v_{3}\right\rangle=\lambda_{1} t_{1}\left\langle v_{1}-v_{2}, t_{1}\left(v_{1}+v_{2}\right)+t_{2} v_{3}\right\rangle=0
$$

and similarly

$$
\left\langle A\left(v_{1}-v_{2}\right), v_{1}-v_{2}\right\rangle=\lambda_{1}\left\langle v_{1}+v_{2}, v_{1}-v_{2}\right\rangle=0 \text {. }
$$

Remark 5.2. The matrix expressions of the $(1,1)$ - and the $(0,2)$-versions of the Cotton-York tensor are different at any point where the matrix for the metric is not the identity. However, the determinant will vanish for one of them if and only if it does for the other.

LCWs in the Thurston geometries. The rest of this section deals with the existence of LCWs among the eight Thurston geometries. A good reference for their definition and properties is the classical paper [Scott 1983]. We begin with the following six geometries:

- $\mathbb{S}^{3}, \mathbb{E}^{3}, \mathbb{H}^{3}$ : These three geometries are conformally flat, and consequently admit multiple LCWs.

- $\mathbb{S}^{2} \times \mathbb{R}, \mathbb{H}^{2} \times \mathbb{R}$ : This case is obvious, with the LCW lying along the $\mathbb{R}$-direction. 
- Sol: Recall that Sol can be seen as $\mathbb{R}^{3}$ with a metric given in the standard coordinates $(x, y, z)$ by

$$
g=e^{2 z} d x^{2}+e^{-2 z} d y^{2}+d z^{2} .
$$

The metric $\bar{g}=e^{-2 z} \cdot g$ splits along $\partial_{x}$, and therefore $g$ has an LCW.

The last two geometries have a different behaviour.

Theorem 5.3. $\widetilde{\mathrm{SL}_{2}(\mathbb{R})}$ and Nil do not admit LCWs.

Proof. We start by recalling the properties we will need.

- $\widetilde{\mathrm{SL}_{2}(\mathbb{R})}$ : Since our study is local, we will work directly in $\mathrm{SL}_{2}(\mathbb{R})$. Being a Lie group, $\mathrm{SL}_{2}(\mathbb{R})$ has a left-invariant metric defined by declaring the three matrices

$$
e_{1}=\left(\begin{array}{rr}
0 & 1 \\
-1 & 0
\end{array}\right), \quad e_{2}=\left(\begin{array}{rr}
\frac{1}{2} & 0 \\
0 & -\frac{1}{2}
\end{array}\right), \quad e_{3}=\left(\begin{array}{ll}
0 & 1 \\
0 & 0
\end{array}\right)
$$

as an orthonormal basis of $T_{I} \mathrm{SL}_{2}(\mathbb{R})$. We will use $E_{1}, E_{2}, E_{3}$ to denote the left-invariant vector fields in $\widetilde{\mathrm{SL}_{2}(\mathbb{R})}$ agreeing with $e_{1}, e_{2}, e_{3}$ at the identity.

To write the metric in coordinates, we will use the Iwasawa decomposition that writes any element in $\mathrm{SL}_{2}(\mathbb{R})$ as an ordered product of three matrices of the form

$$
\left(\begin{array}{rr}
\cos \theta & \sin \theta \\
-\sin \theta & \cos \theta
\end{array}\right), \quad\left(\begin{array}{cc}
e^{t / 2} & 0 \\
0 & e^{-t / 2}
\end{array}\right), \quad\left(\begin{array}{ll}
1 & s \\
0 & 1
\end{array}\right) .
$$

It is easy to see that we can take $\theta, t$ and $s$ as coordinates in a suitable neighbourhood of the identity matrix $I$, with $\partial_{\theta}, \partial_{t}$ and $\partial_{s}$ agreeing with $E_{1}, E_{2}$ and $E_{3}$ at $I$, but not away from it. In fact, in these coordinates, a tedious calculation shows that the coefficients for the above-mentioned left-invariant metric are

$$
\begin{gathered}
g_{\theta \theta}=\left(4 s^{2}+1\right) e^{2 t}+\left(\left(s^{2}-1\right) e^{t}+e^{-t}\right)^{2}, \quad g_{\theta s}=\left(s^{2}-1\right) e^{t}+e^{-t}, \\
g_{\theta t}=\left(\left(s^{2}-1\right) e^{t}+e^{-t}\right) s+2 s e^{t}, \quad g_{t t}=s^{2}+1, \quad g_{t s}=s, \quad g_{s s}=1 .
\end{gathered}
$$

To see this, write the orthonormal basis $\left\{E_{i}\right\}$ in terms of $\partial_{\theta}, \partial_{t}, \partial_{s}$.

Once we have an expression for the metric tensor in coordinates, computing the determinant of the Cotton-York tensor is a matter of following the definitions with a lot of care. The Ricci tensor is

$$
\begin{aligned}
\operatorname{Ric}_{\theta \theta} & =-8 s^{2} e^{2 t}, \\
\operatorname{Ric}_{\theta t} & =\operatorname{Ric}_{t \theta}=-4 s e^{t}, \\
\operatorname{Ric}_{t t} & =-2,
\end{aligned}
$$

the scalar curvature is $s=-2$, the Schouten tensor is

$$
\begin{gathered}
S_{\theta \theta}=-8 s^{2} e^{2 t}+\frac{1}{2}\left(4 s^{2}+1\right) e^{2 t}+\frac{1}{2}\left(\left(s^{2}-1\right) e^{t}+e^{-t}\right)^{2}, \\
S_{\theta t}=\left(\frac{1}{2} s^{3}-\frac{7}{2} s\right) e^{t}+\frac{1}{2} e^{-t} s, \quad S_{\theta s}=\frac{1}{2}\left(s^{2}-1\right) e^{t}+\frac{1}{2} e^{-t}, \\
S_{t t}=-\frac{3}{2}+\frac{1}{2} s^{2}, \quad S_{t s}=\frac{1}{2} s, \quad S_{s s}=\frac{1}{2} .
\end{gathered}
$$


The Cotton-York tensor of $\widehat{\mathrm{SL}_{2}(\mathbb{R})}$ can be computed from these equations and formula (9), yielding

$$
\begin{gathered}
C Y_{\theta \theta}=4 s^{4} e^{2 t}-28 s^{2} e^{(2 t)}+8 s^{2}+8 e^{2 t}+4 e^{(-2 t)}-12, \\
C Y_{\theta t}=4 s^{3} e^{t}+4 s e^{-t}-14 s e^{t}, \quad C Y_{\theta s}=4 s^{2} e^{t}+4 e^{-t}-6 e^{t}, \\
C Y_{t t}=4 s^{2}-4, \quad C Y_{t s}=4 s, \quad C Y_{s s}=4 .
\end{gathered}
$$

When $s=t=0$, this yields

$$
C Y_{(\theta, 0,0)}=\left(\begin{array}{rrr}
0 & 0 & -2 \\
0 & -4 & 0 \\
-2 & 0 & 4
\end{array}\right)
$$

with nonzero determinant. Since the metric is left-invariant, the same happens at any other point.

- Nil: This is the space of triangular matrices of the form

$$
\left\{\left(\begin{array}{lll}
1 & x & z \\
0 & 1 & y \\
0 & 0 & 1
\end{array}\right): x, y, z \in \mathbb{R}\right\},
$$

with the natural left-invariant metric. This turns out to be just $\mathbb{R}^{3}$ with the metric

$$
g=d x^{2}+d y^{2}+(d z-x d y)^{2} .
$$

Once again, we apply the standard formulas, and find the Ricci tensor

$$
\text { Ric }=\left(\begin{array}{ccc}
-\frac{1}{2} & 0 & 0 \\
0 & \frac{1}{2} x^{2}-\frac{1}{2} & -\frac{1}{2} x \\
0 & -\frac{1}{2} x & \frac{1}{2}
\end{array}\right)
$$

the scalar curvature $s=-\frac{1}{2}$, the Schouten tensor,

$$
S=\left(\begin{array}{rcr}
-\frac{3}{8} & 0 & 0 \\
0 & \frac{5}{8} x^{2}-\frac{3}{8} & -\frac{5}{8} x \\
0 & -\frac{5}{8} x & \frac{5}{8}
\end{array}\right),
$$

and the Cotton-York tensor

$$
C Y=\left(\begin{array}{ccr}
\frac{1}{2} & 0 & 0 \\
0 & -x^{2}+\frac{1}{2} & x \\
0 & x & -1
\end{array}\right) .
$$

The determinant of $C Y$ is $-\frac{1}{4}$, and there are no local LCWs in this space.

\section{Proof of Theorem 1.9 in dimensions $n \geqslant 4$}

We divide the proof into two parts. First, we examine the set of algebraic Weyl operators satisfying the eigenflag condition. We prove that this set is semialgebraic (and, in fact, algebraic in dimension 4), and compute its codimension explicitly. Then, we see how to use this to approximate any metric by metrics whose Weyl tensor at a given point $p_{0}$ does not satisfy the eigenflag condition. 
The algebraic part is contained in the following theorem.

Theorem 6.1. The set $\mathcal{E} \mathcal{W}$ of Weyl tensors that satisfy the eigenflag condition is a semialgebraic subset of the space of Weyl tensors with codimension

$$
\frac{1}{3} n^{3}-n^{2}-\frac{4}{3} n+2
$$

In particular, the codimension is 2 for $n=4$ and 12 for $n=5$.

Remark 6.2. A semialgebraic subset of $\mathbb{R}^{n}$ is defined by equations and inequalities involving polynomials. We will need the Tarski-Seidenberg theorem, which states that the image of a semialgebraic set by a map given by polynomials is a semialgebraic set (see [Bochnak et al. 1998, Proposition 2.2.7]). At present, we do not know whether the set of Weyl tensors satisfying the eigenflag condition is an algebraic set; nonetheless, this will not be necessary for the purposes of this paper.

Dimension 4. Before proving Theorem 6.1, we recall the special structure of the Weyl operator in dimension 4 . The curvature tensor in dimension 4 has the following decomposition induced by the Hodge operator $\star($ see Section 2):

$$
R=\left(\begin{array}{cc}
\frac{s}{12} \mathrm{Id}+W^{+} & Z \\
Z^{t} & \frac{s}{12} \mathrm{Id}+W^{-}
\end{array}\right),
$$

where $W^{+}$(resp. $W^{-}$) is any symmetric traceless operator on the 3-dimensional space $\Lambda^{+}\left(\operatorname{resp} . \Lambda^{-}\right)$. Reciprocally, any such operators appear as $W^{+}$and $W^{-}$for some curvature operator.

Clearly there are no simple bivectors in $\Lambda^{+}$or $\Lambda^{-}$. The Weyl operator can have simple eigenvectors only when $W^{+}$and $W^{-}$share some eigenvalue since in that case $W$ could have some eigenspace that would not be contained in $\Lambda^{+}$or $\Lambda^{-}$.

In particular, if all the eigenvalues of $W$ are different, all eigenvectors of $W$ will be nonsimple. This gives the following argument for the density of Weyl operators in dimension 4 that do not satisfy the eigenflag condition.

Let $W_{0}=W_{0}^{+} \oplus W_{0}^{-}$be a Weyl operator in $\mathcal{E} \mathcal{W}$. We define a sequence of Weyl operators $W_{j}$ having the same eigenvectors of $W_{0}$ and such that the corresponding eigenvalues of $W_{j}$ converge to those of $W_{0}$. It is clear that we can choose the six eigenvalues of $W_{j}$ to be different (thus assuring that $W_{j} \notin \mathcal{E} \mathcal{W}$ ) and also such that the three eigenvalues of either $W_{j}^{+}$or $W_{j}^{-}$add up to zero; this assures us that $W_{j}$ is a Weyl operator, thus proving density of the complement of $\mathcal{E} \mathcal{W}$.

Notice that this automatically implies the openness and denseness of the complement of $\mathcal{E}$. Now we turn to the proof of Theorem 6.1.

Proof of Theorem 6.1 for $n=4$. Let $W=W^{+} \oplus W^{-}$be a Weyl operator satisfying the eigenflag condition. Since $W \in \mathcal{E} \mathcal{W}$, there is some $v \in V$ such that $W\left(v \wedge v^{\perp}\right) \subset v \wedge v^{\perp}$. This also implies that $\Lambda^{2}\left(v^{\perp}\right)=\left(v \wedge v^{\perp}\right)^{\perp}$ is an eigenspace of $W$.

We can perform a rotation in $V$ so that $e_{1}=v$ and $e_{1} \wedge e_{2}, e_{1} \wedge e_{3}$ and $e_{1} \wedge e_{4}$ are eigenvectors of the Weyl operator with corresponding eigenvalues $\lambda_{12}, \lambda_{13}$ and $\lambda_{14}$. Notice that the induced rotation in $\Lambda^{2}(V)$ leaves $\Lambda^{+}$and $\Lambda^{-}$invariant. 
We now compute $W\left(e_{3} \wedge e_{4}\right)$ : By the eigenflag condition,

$$
W\left(e_{3} \wedge e_{4}\right) \in\left\langle e_{2} \wedge e_{3}, e_{2} \wedge e_{4}, e_{3} \wedge e_{4}\right\rangle .
$$

By the choice of basis,

$$
W\left(e_{1} \wedge e_{2}+e_{3} \wedge e_{4}\right)=\lambda_{12} e_{1} \wedge e_{2}+W\left(e_{3} \wedge e_{4}\right)
$$

must lie in $\Lambda^{+}$. From $\lambda_{12}\left(e_{1} \wedge e_{2}+e_{3} \wedge e_{4}\right) \in \Lambda^{+}$, it follows that

$$
W\left(e_{1} \wedge e_{2}+e_{3} \wedge e_{4}\right)-\lambda_{12}\left(e_{1} \wedge e_{2}+e_{3} \wedge e_{4}\right) \in\left\langle e_{2} \wedge e_{3}, e_{2} \wedge e_{4}, e_{3} \wedge e_{4}\right\rangle \cap \Lambda^{+}=\{0\} .
$$

Hence $W\left(e_{3} \wedge e_{4}\right)=\lambda_{12} e_{3} \wedge e_{4}$. Similarly, $W\left(e_{2} \wedge e_{4}\right)=\lambda_{13} e_{2} \wedge e_{4}$ and $W\left(e_{2} \wedge e_{3}\right)=\lambda_{14} e_{2} \wedge e_{3}$.

Thus in the basis of $\Lambda^{2}(V)$ as given in (10) and (11), $W$ is written as

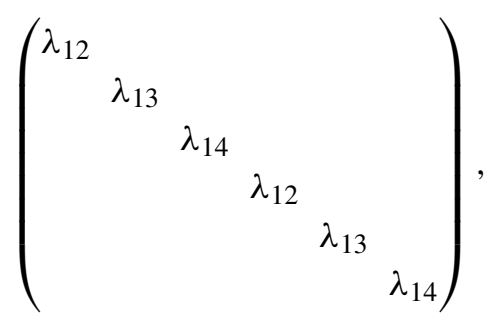

and since both $W^{+}$and $W^{-}$are traceless, $\lambda_{12}+\lambda_{13}+\lambda_{14}=0$.

The dimension of the space of Weyl tensors in dimension 4 is 10. Let us now compute the dimension of $\mathcal{E W}$. By the above, the map

$$
\Phi: \mathrm{SO}(V) \times \mathbb{R}^{2} \rightarrow \mathcal{E} \mathcal{W},
$$

sending $\left(\rho, \lambda_{12}, \lambda_{13}\right)$ to

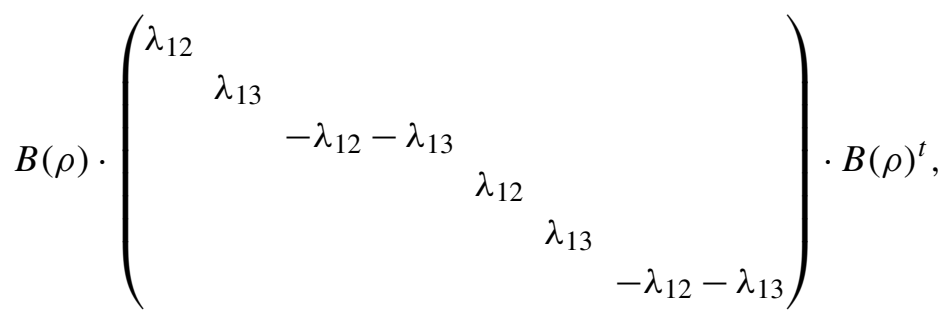

is surjective, where $B(\rho)$ is the rotation on $\Lambda^{2}(V)$ induced by $\rho$.

This means that $\mathcal{E W}$ is the image of an algebraic set by an algebraic map, so it is a semialgebraic subset of $\mathcal{W}$ by the Tarski-Seidenberg theorem [Bochnak et al. 1998, Proposition 2.2.7]. The map is singular only if two of the three numbers $\lambda_{12}, \lambda_{13}$ and $\lambda_{14}=-\lambda_{12}-\lambda_{13}$ coincide, or if all of them vanish. This implies that the map $\Phi$ is locally injective in an open set, and thus the dimension of $\mathcal{E W}$ is $\operatorname{dim} \mathrm{SO}(V)+2=8$.

Remark 6.3. As mentioned before, we do not know whether $\mathcal{E W}$ is an algebraic set. However, in dimension 4, we have shown that operators in $\mathcal{E} \mathcal{W}$ have at least one double eigenvalue. It follows that $\mathcal{E} \mathcal{W}$ is contained in a proper algebraic set. 
Theorem 6.4. In dimension 4, the set of Weyl tensors having different eigenvalues and nonsimple eigenvectors is the complement of a proper algebraic set.

Proof. The set of algebraic operators with at least one multiple eigenvalue is an algebraic set given by the equations

$$
\Delta_{t}(\operatorname{det}(t W-I))=0
$$

where $\Delta_{t}$ is the discriminant of a polynomial in $t$. The discriminant of the characteristic polynomial of $W$ vanishes exactly when the characteristic polynomial has nonsimple roots, which happens when the operator has eigenspaces of dimension greater than 1 .

\section{Weyl tensors with the eigenflag condition in dimensions $n \geqslant 5$.}

Proof of Theorem 6.1 for $n \geqslant 5$. As in dimension 4, we will find an algebraic map from a space of dimension smaller than $\operatorname{dim} \mathcal{W}$ whose image is exactly $\mathcal{E} \mathcal{W}$ and use [Bochnak et al. 1998, Proposition 2.2.7] to show that $\mathcal{E} \mathcal{W}$ is semialgebraic.

Let $W$ be an algebraic Weyl operator with the eigenflag condition on the vector space $V$. We will build an orthonormal basis of $V$ such that $W$ is written conveniently.

By hypothesis, there is vector $v$ such that $W\left(v \wedge v^{\perp}\right) \subset v \wedge v^{\perp}$. The operator $\left.W\right|_{v \wedge v^{\perp}}$ is symmetric and diagonalizes in an orthonormal basis of bivectors contained in $v \wedge v^{\perp}$. All such eigenvectors are of the form $v \wedge w$, and two such bivectors $v \wedge w_{1}$ and $v \wedge w_{2}$ are orthogonal if and only if $w_{1}$ is orthogonal to $w_{2}$. We let $\left\{e_{1}=v, e_{2}, \ldots, e_{n}\right\}$ be an orthonormal basis of $v \wedge v^{\perp}$ such that $\left.W\right|_{v \wedge v^{\perp}}$ is diagonal in the basis $e_{1} \wedge e_{k}$, with eigenvalue $\lambda_{k}$.

Then, in this basis,

$$
W=\left(\begin{array}{llll}
\lambda_{2} & & & \\
& \ddots & & \\
& & \lambda_{n} & \\
& & & W_{2}
\end{array}\right) .
$$

In other words,

$$
W=\sum \lambda_{k} e_{1 k} \odot e_{1 k}+W_{2},
$$

where $W_{2}$ is a symmetric operator on the vector space $\Lambda^{2}\left(v^{\perp}\right)$ and $e_{a b} \odot e_{c d}$ denotes the symmetric endomorphism of $\Lambda^{2} V$ sending $e_{a} \wedge e_{b}$ to $e_{c} \wedge e_{d}$ and vice versa; notice that we will use the same $\odot$ notation to indicate also the symmetric product in $V$; it will be clear from the context which situation applies.

Notice that

$$
b(W)=0, \quad b\left(e_{1 k} \odot e_{1 k}\right)=0,
$$

where $b$ is the Bianchi projector defined as in (3); we obtain that $W_{2}$ is a curvature operator. It may not be a Weyl operator, because for the Ricci projector $r$ introduced in (4),

$$
r\left(e_{1 k} \odot e_{1 k}\right)=e_{1} \odot e_{1}+e_{k} \odot e_{k} .
$$


Nonetheless, we can deduce that $\sum_{k=2}^{n} \lambda_{k}=0$ because

$$
0=\left\langle r(W), e_{1} \odot e_{1}\right\rangle=\sum_{k=2}^{n} \lambda_{k}\left\langle r\left(e_{1 k} \odot e_{1 k}\right), e_{1} \odot e_{1}\right\rangle+\left\langle r\left(W_{2}\right), e_{1} \odot e_{1}\right\rangle,
$$

and $\left\langle r\left(W_{2}\right), e_{1} \odot e_{1}\right\rangle=0$ because $W_{2}$ is an operator on the orthogonal complement of $e_{1}$. Together with (16),

$$
r\left(W_{2}\right)=-\sum_{k=2}^{n} \lambda_{k} r\left(e_{1 k} \odot e_{1 k}\right)=-\left(\sum_{k=2}^{n} \lambda_{k} e_{k} \odot e_{k}\right) .
$$

In other words, $W_{2} \in \operatorname{ker}(b) \cap r^{-1}\left(-\sum_{k=2}^{n} \lambda_{k} e_{k} \odot e_{k}\right)$. We denote this (affine) space by $\mathcal{R}\left(\left\{\lambda_{k}\right\}\right)$; its dimension will agree with the dimension of $\mathcal{W}\left(v^{\perp}\right)=\operatorname{ker}(b) \cap \operatorname{ker}(r)$.

Hence if $W \in \mathcal{E} \mathcal{W}$, there exist an element $\rho \in \operatorname{SO}(V)$, numbers $\lambda_{2}, \ldots, \lambda_{n}$ with $\sum_{k} \lambda_{k}=0$, and a curvature operator $W_{2} \in \mathcal{R}\left(\left\{\lambda_{k}\right\}\right)$ such that

$$
W=B(\rho) \cdot\left(\sum \lambda_{k} e_{1 k} \odot e_{1 k}+\left(\begin{array}{cccc}
0 & & & \\
& \ddots & & \\
& & 0 & \\
& & & W_{2}
\end{array}\right)\right) \cdot B(\rho)^{t},
$$

where remember that $B(\rho)$ is the map in bivectors induced by $\rho$. Let

$$
\mathbb{S}=\left\{\left(\lambda_{k}\right)_{k=2, \ldots, n}: \sum \lambda_{k}=0\right\}
$$

and define a map

$$
\Phi: \operatorname{SO}(V) \times \mathbb{S} \times \mathcal{R}\left(\left\{\lambda_{k}\right\}\right) \rightarrow \mathcal{W}
$$

by the above formula (18).

We know that

$$
\sum \lambda_{k} e_{1 k} \odot e_{1 k}+\left(\begin{array}{cccc}
0 & & & \\
& \ddots & & \\
& & 0 & \\
& & & W_{2}
\end{array}\right)
$$

is a Weyl tensor because it lies in the kernel of $b$ and $r$, and conjugating by $B(\rho)$ produces another Weyl tensor by equation (5). It follows that $\Phi\left(\rho,\left\{\lambda_{k}\right\}, W_{2}\right)$ is always a Weyl tensor, and it is clear that it has the eigenflag property. Thus $\Phi$ is surjective onto $\mathcal{E} \mathcal{W}$.

We will now compute the dimension of $\mathcal{E} \mathcal{W}$. The dimension of the space of curvature operators is

$$
\operatorname{dim} \mathcal{R}_{n}=\operatorname{dim} S^{2}\left(\Lambda^{2} V\right)-\operatorname{dim}\left(\Lambda^{4} V\right)=\frac{1}{12} n^{4}-\frac{1}{12} n^{2}
$$

The dimension of the space of Weyl operators is

$$
\operatorname{dim} \mathcal{W}_{n}=\operatorname{dim} \mathcal{R}_{n}-\operatorname{dim} S^{2}(V)=\frac{1}{12} n^{4}-\frac{7}{12} n^{2}-\frac{1}{2}
$$

The dimension of $\mathrm{SO}(V) \times \mathbb{S} \times \mathcal{R}\left(\left\{\lambda_{k}\right\}\right)$ is thus the sum of

$$
\operatorname{dim} \operatorname{SO}(V)=\left(\begin{array}{l}
n \\
2
\end{array}\right), \quad \operatorname{dim} \mathbb{S}=n-2, \quad \operatorname{dim} \mathcal{R}\left(\left\{\lambda_{k}\right\}\right)=\frac{1}{12}(n-1)^{4}-\frac{7}{12}(n-1)^{2}-\frac{1}{2}
$$


However, the dimension of $\mathrm{SO}(V) \times \mathbb{S} \times \mathcal{R}\left(\left\{\lambda_{k}\right\}\right)$ could be strictly greater than that of $\mathcal{E} \mathcal{W}$. In order to prove that this is not the case, we show that $\Phi$ is finite-to-one when restricted to a nontrivial open subset $\mathcal{A}$ of $\mathrm{SO}(V) \times \mathbb{S} \times \mathcal{R}\left(\left\{\lambda_{k}\right\}\right)$.

Let $w$ be the projection from the curvature operators onto the Weyl tensors. Then $\mathcal{A}$ is the set of triples $\left(\rho,\left\{\lambda_{k}\right\}, R\right)$ such that

- all $\lambda_{k}$ for $k=2, \ldots, n$ are different,

- the Weyl tensor $w(R)$ does not satisfy the eigenflag condition.

It is clear that $\mathcal{A}$ is open. In order to see that it is not empty, we use induction to find a Weyl tensor $W_{2}$ on the space $\partial_{1}^{\perp}$ that does not satisfy the eigenflag condition. The base case for the induction is dimension 4 , which was done in the previous section. We fix arbitrary $\left\{\lambda_{k}\right\}$ whose sum is 0 , and choose any rotation $\rho$. Let $R_{0}$ be any operator in $\mathcal{R}\left(\left\{\lambda_{k}\right\}\right)$. Then $R_{1}=R_{0}+W_{2}-w\left(R_{0}\right)$ is a curvature operator in the affine space $\mathcal{R}\left(\left\{\lambda_{k}\right\}\right)$ whose projection $w\left(R_{1}\right)$ to the space of Weyl tensors is $W_{2}$.

For $W \in \Phi(\mathcal{A})$, let us compute its preimages $\left(\rho,\left\{\lambda_{k}\right\}, R_{n-1}\right)$ in $\mathcal{A}$. The direction $v_{1}$ is a direction with the eigenflag property, and by the hypothesis, it is unique up to sign. The numbers $\lambda_{k}$ for $k=2, \ldots, n$ are the unique eigenvalues of $\left.W\right|_{v_{1} \wedge v_{1}^{\perp}}$, up to change of order. The $v_{k}$ are unit-vectors in $v_{1}^{\perp}$ such that $v_{1} \wedge v_{k}$ are eigenvectors of $\left.W\right|_{v_{1} \wedge v_{1}^{\perp}}$ corresponding to the eigenvalues $\lambda_{k}$, and they are unique up to a change of sign. The basis $v_{k}$ determines $\rho$ uniquely and $R_{n-1}$ is the unique remainder $B(\rho)^{t} \circ W \circ B(\rho)-\sum \lambda_{k} e_{1 k} \odot e_{1 k}$. It follows that $\Phi^{-1}(W)$ is finite for any $W$, and $\operatorname{dim}(\mathcal{E} \mathcal{W})$ agrees with $\operatorname{dim}\left(\operatorname{SO}(V) \times \mathbb{S} \times \mathcal{R}\left(\left\{\lambda_{k}\right\}\right)\right)$. Thus using the above formulae, we obtain that the codimension of $\mathcal{E} \mathcal{W}$ inside $\mathcal{W}$ is

$$
\frac{1}{3} n^{3}-n^{2}-\frac{4}{3} n+2 .
$$

Proof of Theorem 1.9 for $n=\operatorname{dim} \boldsymbol{M} \geqslant 4$. We start with a precise statement of a folklore lemma in Riemannian geometry.

Lemma 6.5. Let $M$ be a Riemannian manifold with metric $g$ and $p$ any point in $M$, with $R(p)$ the curvature of the metric $g$ at $p$.

Then for any algebraic curvature operator $R^{0}$ close enough to $R(p)$, there exists a metric $g^{\prime}$ that agrees with $g$ outside a neighbourhood of $p$ and such that the curvature of $g^{\prime}$ at $p$ is $R^{0}$.

Furthermore, we can choose $g^{\prime}$ such that

$$
\left\|g^{\prime}-g\right\|_{C^{2}} \leqslant C\left\|R^{0}-R(p)\right\|,
$$

with a constant $C$ independent of $R^{0}$.

Remark 6.6. The norm appearing in the left-hand side in the above inequality is computed in a fixed set of coordinates of $p$.

Proof. We use the following formula for the computation of the Riemannian curvature in terms of partial derivatives of $g$ and the Christoffel symbols:

$$
R_{i k \ell m}=\frac{1}{2}\left(\frac{\partial^{2} g_{i m}}{\partial x^{k} \partial x^{\ell}}+\frac{\partial^{2} g_{k \ell}}{\partial x^{i} \partial x^{m}}-\frac{\partial^{2} g_{i \ell}}{\partial x^{k} \partial x^{m}}-\frac{\partial^{2} g_{k m}}{\partial x^{i} \partial x^{\ell}}\right)+g_{n p}\left(\Gamma_{k \ell}^{n} \Gamma_{i m}^{p}-\Gamma_{k m}^{n} \Gamma_{i \ell}^{p}\right) .
$$


Take normal coordinates for the metric $g$ at $p$. In these coordinates, the Christoffel symbols at $p$ vanish.

In these coordinates, choose a smooth function $\varphi$ with value 1 near $p$ and value 0 in the complement of the domain of the coordinates. Define a new metric as

$$
g_{i j}^{\prime}=g_{i j}-\frac{1}{4} \sum_{k, h} R_{i h j k}^{*} x^{h} x^{k} \varphi(x)
$$

in the coordinate patch, and by $g$ outside of it, where $R^{*}=R^{0}-R(p)$. If $R^{*}$ is small enough, $g^{\prime}$ will still be positive definite. The Christoffel symbols are given by

$$
\Gamma_{i j}^{m}=\frac{1}{2} g^{m k}\left(\frac{\partial}{\partial x^{j}} g_{k i}+\frac{\partial}{\partial x^{i}} g_{k j}-\frac{\partial}{\partial x^{k}} g_{i j}\right) .
$$

Thus, since the Christoffel symbols of $g$ vanish, and we have added a quadratic perturbation to $g$, the Christoffel symbols of $g^{\prime}$ also vanish. We compute the curvature of $g^{\prime}$ at $p$ using (19):

$$
R^{\prime}(p)_{i k l m}=R(p)_{i k l m}-\frac{1}{4}\left(R_{i k m l}^{*}+R_{k i l m}^{*}-R_{i k l m}^{*}-R_{k i m l}^{*}\right)=R(p)_{i k l m}+R_{i k l m}^{*}=R_{i k l m}^{0} .
$$

The $C^{2}$-norm of $g^{\prime}-g$ is bounded by $C\left\|R^{*}\right\|$, with a constant $C$ independent of $R^{*}$.

Proof of Theorem 1.9 for $\operatorname{dim} M \geqslant 4$. Let $U \subset M$ for a compact manifold $M$. Denote by $\mathcal{O}$ the set of Riemannian metrics on $M$ for which there is at least one point $p \in U$ such that the Weyl tensor $W_{p}$ of $g$ at $p$ does not satisfy the eigenflag condition. By Theorem $1.3, \mathcal{O}$ is contained in the set of metrics that do not admit an LCW on $U$.

Since the complement of $\mathcal{E} \mathcal{W}$ is open, and the map that assigns its Weyl tensor to a Riemannian metric is continuous under $C^{2}$-deformations of the metric, $\mathcal{O}$ is open.

For density, fix an arbitrary point $p_{0} \in U$ and consider a metric $g$ such that $W(g)_{p_{0}} \in \mathcal{E} \mathcal{W}$. By Theorem 6.1, we can find a Weyl tensor $\widetilde{W} \notin \mathcal{E} \mathcal{W}$ such that $\left\|\widetilde{W}-W(g)_{p_{0}}\right\|<\epsilon$.

We choose $R_{0}=R(g)_{p_{0}}-W(g)_{p_{0}}+\widetilde{W}$ and apply Lemma 6.5 to get a new metric $g^{\prime}$ that satisfies $\left\|g^{\prime}-g\right\|_{C^{2}} \leqslant C\left\|\widetilde{W}-W(g)_{p_{0}}\right\|<C \epsilon$. The Weyl tensor of $g^{\prime}$ at $p_{0}$ is $\widetilde{W} \notin \mathcal{E} \mathcal{W}$; thus $g^{\prime}$ is not in $\mathcal{O}$. Since $\epsilon$ is arbitrary, denseness of $\mathcal{O}$ follows.

Proof of Theorem 1.9 for $\boldsymbol{n}=\operatorname{dim} \boldsymbol{M}=3$. In this section, we use the Cotton tensor instead of the Weyl tensor.

The space of algebraic Cotton-York tensors at $p \in M$ consists of simply the symmetric, traceless operators on the euclidean space $T_{p} M$. It is obvious that the set of Cotton-York tensors with zero determinant is a proper algebraic subset of the set of all such tensors.

The following result is the equivalent of Lemma 6.5 for the Cotton tensor:

Lemma 6.7. Let $M$ be a Riemannian manifold with metric $g$ and $p$ any point in $M$.

Then for any algebraic Cotton-York tensor $C Y^{0}$ close enough to $C Y_{p}$, we can find a metric $g^{\prime}$ that agrees with $g$ outside a neighbourhood of $p$ so that the Cotton-York tensor of $g^{\prime}$ at $p$ is $C Y^{0}$.

Furthermore, we can find the metric $g^{\prime}$ in such a way that the $C^{3}$-norm of $\left|g-g^{\prime}\right|$ is bounded by a multiple of the norm of $C Y^{0}-C Y_{p}$. 
Proof. Our first goal is to find a formula that expresses the Cotton tensor at $p$ in terms of the metric tensor and its derivatives. Take normal coordinates at $p$, so that $g_{p}$ is the identity matrix, and the Christoffel symbols vanish at $p$. We start with the formula (19) for the curvature tensor and take derivatives.

We compute first the Schouten tensor in a neighbourhood of $p$ :

$$
S_{a b}=\frac{1}{2}\left(\delta_{i a} \delta_{l b}-\frac{1}{4} g_{a b} g^{i l}\right) g^{k m}\left(\frac{\partial^{2} g_{i m}}{\partial x^{k} \partial x^{\ell}}+\frac{\partial^{2} g_{k \ell}}{\partial x^{i} \partial x^{m}}-\frac{\partial^{2} g_{i \ell}}{\partial x^{k} \partial x^{m}}-\frac{\partial^{2} g_{k m}}{\partial x^{i} \partial x^{\ell}}\right)+Q(\Gamma),
$$

where $Q(\Gamma)$ consists of terms like $\Gamma_{k \ell}^{n} \Gamma_{i m}^{p}$.

The covariant derivative $\nabla_{n} S_{a b}(p)=\left(\partial / \partial x^{n}\right) S_{a b}(p)$ at $p$ is

$$
\nabla_{n} S_{a b}(p)=\frac{1}{2} \frac{\partial}{\partial x^{n}}\left(\frac{\partial^{2} g_{a k}}{\partial x^{k} \partial x^{b}}+\frac{\partial^{2} g_{k b}}{\partial x^{a} \partial x^{k}}-\frac{\partial^{2} g_{a b}}{\partial x^{k} \partial x^{k}}-\frac{\partial^{2} g_{k k}}{\partial x^{a} \partial x^{b}}\right)-\frac{1}{4} \frac{\partial}{\partial x^{n}}\left(\frac{\partial^{2} g_{i k}}{\partial x^{k} \partial x^{i}}-\frac{\partial^{2} g_{k k}}{\partial x^{i} \partial x^{i}}\right) \delta_{a b} .
$$

The derivatives of $Q(\Gamma)$ vanish because one Christoffel symbol will remain in the final computation, and it evaluates to 0 at $p$.

The Cotton tensor at $p$ is

$$
\begin{aligned}
& C_{n a b}(p) \\
& \begin{aligned}
=\frac{1}{2} & \frac{\partial}{\partial x^{n}}\left(\frac{\partial^{2} g_{a k}}{\partial x^{k} \partial x^{b}}+\frac{\partial^{2} g_{k b}}{\partial x^{a} \partial x^{k}}-\frac{\partial^{2} g_{a b}}{\partial x^{k} \partial x^{k}}-\frac{\partial^{2} g_{k k}}{\partial x^{a} \partial x^{b}}\right)-\frac{1}{2} \frac{\partial}{\partial x^{a}}\left(\frac{\partial^{2} g_{n k}}{\partial x^{k} \partial x^{b}}+\frac{\partial^{2} g_{k b}}{\partial x^{n} \partial x^{k}}-\frac{\partial^{2} g_{n b}}{\partial x^{k} \partial x^{k}}-\frac{\partial^{2} g_{k k}}{\partial x^{n} \partial x^{b}}\right) \\
& \quad-\frac{1}{4} \frac{\partial}{\partial x^{n}}\left(\frac{\partial^{2} g_{i k}}{\partial x^{k} \partial x^{i}}-\frac{\partial^{2} g_{k k}}{\partial x^{i} \partial x^{i}}\right)+\delta_{a b} \frac{1}{4} \frac{\partial}{\partial x^{a}}\left(\frac{\partial^{2} g_{i k}}{\partial x^{k} \partial x^{i}}-\frac{\partial^{2} g_{k k}}{\partial x^{i} \partial x^{i}}\right) \delta_{n b} \\
= & \frac{1}{2}\left(\frac{\partial^{3} g_{a k}}{\partial x^{k} \partial x^{n} \partial x^{b}}-\frac{\partial^{3} g_{n k}}{\partial x^{k} \partial x^{a} \partial x^{b}}-\frac{\partial^{3} g_{a b}}{\partial x^{k} \partial x^{n} \partial x^{k}}+\frac{\partial^{3} g_{n b}}{\partial x^{k} \partial x^{a} \partial x^{k}}\right)-\frac{1}{4}\left(\frac{\partial^{3} g_{i k}}{\partial x^{k} \partial x^{i} \partial x^{n}}-\frac{\partial^{2} g_{k k}}{\partial x^{i} \partial x^{i} \partial x^{n}}\right) \delta_{a b} \\
& +\frac{1}{4}\left(\frac{\partial^{3} g_{i k}}{\partial x^{k} \partial x^{i} \partial x^{a}}-\frac{\partial^{2} g_{k k}}{\partial x^{i} \partial x^{i} \partial x^{a}}\right) \delta_{n b .} .
\end{aligned}
\end{aligned}
$$

If the $A_{i j}^{k l m}$ are small enough real numbers, symmetric under permutations of $i, j$ and also under permutations of $k, l, m$ (there are 60 different such terms), then

$$
g_{i j}^{\prime}=g_{i j}+\sum A_{i j}^{k l m} x^{k} x^{l} x^{m}
$$

defines a new metric $g^{\prime}$.

The new Cotton tensor at 0 is

$$
C_{n a b}^{\prime}(p)=C_{n a b}(p)+\frac{1}{2}\left(A_{k a}^{k n b}-A_{k n}^{k a b}-A_{a b}^{k k n}+A_{n b}^{k k a}\right)-\frac{1}{4}\left(A_{k i}^{k i n}-A_{k k}^{i i n}\right) \delta_{a b}+\frac{1}{4}\left(A_{k i}^{k i a}-A_{k k}^{i i a}\right) \delta_{n b} .
$$

We define $A$ to be the real vector space of dimension 60 whose coordinates are indexed by the tuples $(\{i, j\},\{k, l, m\})$. The formula

$$
A_{i j}^{m l k} \stackrel{L}{\longrightarrow} \frac{1}{2}\left(A_{k a}^{k n b}-A_{k n}^{k a b}-A_{a b}^{k k n}+A_{n b}^{k k a}\right)-\frac{1}{4}\left(A_{k i}^{k i n}-A_{k k}^{i i n}\right) \delta_{a b}+\frac{1}{4}\left(A_{k i}^{k i a}-A_{k k}^{i i a}\right) \delta_{n b}
$$


defines a linear map $L: \mathbb{A} \rightarrow \mathcal{C}_{p}$ into the space of algebraic Cotton tensors (the $(0,3)$-tensors with the symmetries (8)). It follows from (24) that the image of $L$ consists of Cotton tensors, but it is a nice exercise to check it directly.

In order to show that we can prescribe the Cotton tensor at $p$, we just need to check that $L$ is surjective. The map from the Cotton tensors to the Cotton-York tensors is a linear isomorphism, so we only need to check that the image of the above linear map has dimension 5. Let $L\left(e_{i j}^{k l m}\right)$ be the image by $L$ of the basis vector $e_{i j}^{k l m} \in \mathbb{A}$, with $A_{i j}^{k m l}=1$ and the other entries equal to 0 . The reader may check, for instance, that $L\left(e_{11}^{122}\right), L\left(e_{11}^{123}\right), L\left(e_{11}^{222}\right), L\left(e_{11}^{223}\right)$ and $L\left(e_{12}^{223}\right)$ are linearly independent.

Proof of Theorem 1.9 for $\operatorname{dim} M=3$. Let $U \subset M$ for a compact manifold $M$. This time, $\mathcal{O}$ is the set of Riemannian metrics on $M$ for which there is at least one point $p \in U$ such that the Cotton-York tensor $C Y_{p}$ of $g$ at $p$ has nonzero determinant. By Theorem 1.6, $\mathcal{O}$ is contained in the set of metrics that do not admit an LCW on $U$.

Since the map that assigns its Cotton tensor to a Riemannian metric is continuous under $C^{3}$-deformations of the metric, $\mathcal{O}$ is open in the $C^{3}$-topology.

For density, let $\epsilon>0$, fix an arbitrary point $p_{0} \in U$ and consider a metric $g$ such that its CottonYork tensor $C Y(g)_{p_{0}}$ at $p_{0}$ has zero determinant. Choose a symmetric traceless tensor with nonzero determinant $C Y^{0}$ and such that $\left\|C Y^{0}-C Y(g)_{p_{0}}\right\|<\epsilon$.

We apply Lemma 6.5 to get a new metric $g^{\prime}$ that satisfies $\left\|g^{\prime}-g\right\|_{C^{3}} \leqslant C\left\|C Y^{0}-C Y(g)_{p_{0}}\right\|<C \epsilon$ and whose Cotton-York tensor at $p_{0}$ is $C Y^{0}$. It follows that $g^{\prime}$ is not in $\mathcal{O}$, and since $\epsilon$ is arbitrary, we deduce that $\mathcal{O}$ is dense.

\section{Acknowledgment}

We thank an anonymous referee for a careful reading of the first version of this paper and for pointing out some corrections.

\section{References}

[Angulo-Ardoy 2015] P. Angulo-Ardoy, "On the set of metrics without local limiting Carleman weights", preprint, 2015. arXiv 1509.02127

[Besse 1987] A. L. Besse, Einstein manifolds, Ergebnisse der Mathematik und ihrer Grenzgebiete (3) 10, Springer, Berlin, 1987. MR 867684 Zbl 0613.53001

[Bochnak et al. 1998] J. Bochnak, M. Coste, and M.-F. Roy, Real algebraic geometry, Ergebnisse der Mathematik und ihrer Grenzgebiete (3) 36, Springer, Berlin, 1998. MR 1659509 Zbl 0912.14023

[Calderón 1980] A.-P. Calderón, "On an inverse boundary value problem”, pp. 65-73 in Seminar on numerical analysis and its applications to continuum physics (Rio de Janeiro, 1980), vol. 12, Sociedade Brasileira de Matemática, Rio de Janeiro, 1980. MR 590275 Zbl 1182.35230

[do Carmo 1992] M. P. do Carmo, Riemannian geometry, Birkhäuser, Boston, MA, 1992. MR 1138207 Zbl 0752.53001

[Caro and Salo 2014] P. Caro and M. Salo, "Stability of the Calderón problem in admissible geometries", Inverse Probl. Imaging 8:4 (2014), 939-957. MR 3295953 Zbl 1328.35302

[Cheeger and Ebin 1975] J. Cheeger and D. G. Ebin, Comparison theorems in Riemannian geometry, North-Holland Mathematical Library 9, North-Holland, Amsterdam, 1975. MR 0458335 Zbl 0309.53035 
[Dos Santos Ferreira et al. 2009] D. Dos Santos Ferreira, C. E. Kenig, M. Salo, and G. Uhlmann, "Limiting Carleman weights and anisotropic inverse problems", Invent. Math. 178:1 (2009), 119-171. MR 2534094 Zbl 1181.35327

[Dos Santos Ferreira et al. 2013a] D. Dos Santos Ferreira, C. E. Kenig, and M. Salo, "Determining an unbounded potential from Cauchy data in admissible geometries", Comm. Partial Differential Equations 38:1 (2013), 50-68. MR 3005546 Zbl 1302.35436

[Dos Santos Ferreira et al. 2013b] D. Dos Santos Ferreira, Y. Kurylev, M. Lassas, and M. Salo, "The Calderón problem in transversally anisotropic geometries", preprint, 2013. To appear in J. Eur. Math. Soc. (JEMS). arXiv 1305.1273

[Hertrich-Jeromin 2003] U. Hertrich-Jeromin, Introduction to Möbius differential geometry, London Mathematical Society Lecture Note Series 300, Cambridge University Press, 2003. MR 2004958 Zbl 1040.53002

[Kenig et al. 2011] C. E. Kenig, M. Salo, and G. Uhlmann, "Reconstructions from boundary measurements on admissible manifolds", Inverse Probl. Imaging 5:4 (2011), 859-877. MR 2852376 Zbl 1237.35160

[Kohn and Vogelius 1984] R. V. Kohn and M. Vogelius, "Determining conductivity by boundary measurements", Comm. Pure Appl. Math. 37:3 (1984), 289-298. MR 739921 Zbl 0586.35089

[Kohn and Vogelius 1985] R. V. Kohn and M. Vogelius, "Determining conductivity by boundary measurements, II: Interior results”, Comm. Pure Appl. Math. 38:5 (1985), 643-667. MR 803253 Zbl 0595.35092

[Liimatainen and Salo 2012] T. Liimatainen and M. Salo, "Nowhere conformally homogeneous manifolds and limiting Carleman weights", Inverse Probl. Imaging 6:3 (2012), 523-530. MR 2974608 Zbl 1257.53056

[Liimatainen and Salo 2015] T. Liimatainen and M. Salo, "Local gauge conditions for ellipticity in conformal geometry", Int. Math. Res. Not. (online publication October 2015).

[Sakai 1996] T. Sakai, Riemannian geometry, Translations of Mathematical Monographs 149, American Mathematical Society, Providence, RI, 1996. MR 1390760 Zbl 0886.53002

[Salo 2013] M. Salo, "The Calderón problem on Riemannian manifolds", pp. 167-247 in Inverse problems and applications: inside out, II (Berkeley, CA, 2010), edited by G. Uhlmann, Mathematical Sciences Research Institute Publications 60, Cambridge University Press, 2013. MR 3098658 Zbl 1316.35301

[Scott 1983] P. Scott, "The geometries of 3-manifolds", Bull. London Math. Soc. 15:5 (1983), 401-487. MR 705527 Zbl 0561.57001

[York 1971] J. W. York, Jr., "Gravitational degrees of freedom and the initial-value problem", Phys. Rev. Lett. 26 (1971), 1656-1658. MR 0290765

Received 10 Sep 2014. Revised 15 Jun 2015. Accepted 30 Jan 2016.

PABlo AngulO-ARDoY: pablo.angulo@uam.es

Department of Mathematics, Universidad Autónoma de Madrid, 28049 Madrid, Spain

DANIEL FARACO: daniel.faraco@uam.es

Department of Mathematics, Universidad Autónoma de Madrid, 28049 Madrid, Spain

and

Instituto de Ciencias Matemáticas CSIC-UAM-UCM-UC3M, 28049 Madrid, Spain

LUIS GUIJARRO: luis.guijarro@uam.es

Department of Mathematics, Universidad Autónoma de Madrid, 28049 Madrid, Spain

and

Instituto de Ciencias Matemáticas CSIC-UAM-UCM-UC3M, 28049 Madrid, Spain

ALBERTo RUIZ: alberto.ruiz@uam.es

Department of Mathematics, Universidad Autónoma de Madrid, 28049 Madrid, Spain

and

Instituto de Ciencias Matemáticas CSIC-UAM-UCM-UC3M, 28049 Madrid, Spain 


\title{
Analysis \& PDE
}

msp.org/apde

\section{EDITORS}

\author{
EDITOR-IN-CHIEF \\ Patrick Gérard \\ patrick.gerard@math.u-psud.fr \\ Université Paris Sud XI \\ Orsay, France
}

BOARD OF EDITORS

Nicolas Burq Université Paris-Sud 11, France nicolas.burq@math.u-psud.fr

Massimiliano Berti Scuola Intern. Sup. di Studi Avanzati, Italy berti@sissa.it

Sun-Yung Alice Chang Princeton University, USA chang@math.princeton.edu

Michael Christ University of California, Berkeley, USA mchrist@math.berkeley.edu

Charles Fefferman Princeton University, USA cf@math.princeton.edu

Ursula Hamenstaedt Universität Bonn, Germany ursula@math.uni-bonn.de

Vaughan Jones U.C. Berkeley \& Vanderbilt University vaughan.f.jones@vanderbilt.edu

Vadim Kaloshin University of Maryland, USA vadim.kaloshin@gmail.com

Herbert Koch Universität Bonn, Germany koch@math.uni-bonn.de

Izabella Laba University of British Columbia, Canada ilaba@math.ubc.ca

Gilles Lebeau Université de Nice Sophia Antipolis, France lebeau@unice.fr

László Lempert Purdue University, USA lempert@math.purdue.edu

Richard B. Melrose Massachussets Inst. of Tech., USA rbm@math.mit.edu

Frank Merle Université de Cergy-Pontoise, France Frank.Merle@u-cergy.fr

William Minicozzi II Johns Hopkins University, USA minicozz@math.jhu.edu

Clément Mouhot Cambridge University, UK c.mouhot@dpmms.cam.ac.uk
Werner Müller

Yuval Peres

Gilles Pisier

Tristan Rivière

Igor Rodnianski

Wilhelm Schlag

Sylvia Serfaty

Yum-Tong Siu

Terence Tao

Michael E. Taylor

Gunther Uhlmann

András Vasy

Dan Virgil Voiculescu

Steven Zelditch

Maciej Zworski
Universität Bonn, Germany mueller@math.uni-bonn.de

University of California, Berkeley, USA peres@stat.berkeley.edu

Texas A\&M University, and Paris 6 pisier@math.tamu.edu

ETH, Switzerland riviere@math.ethz.ch Princeton University, USA irod@math.princeton.edu University of Chicago, USA schlag@math.uchicago.edu New York University, USA serfaty@cims.nyu.edu Harvard University, USA siu@math.harvard.edu University of California, Los Angeles, USA tao@math.ucla.edu Univ. of North Carolina, Chapel Hill, USA met@math.unc.edu

University of Washington, USA gunther@math.washington.edu

Stanford University, USA andras@math.stanford.edu

University of California, Berkeley, USA dvv@math.berkeley.edu

Northwestern University, USA zelditch@math.northwestern.edu

University of California, Berkeley, USA zworski@math.berkeley.edu

PRODUCTION

production@msp.org

Silvio Levy, Scientific Editor

See inside back cover or msp.org/apde for submission instructions.

The subscription price for 2016 is US \$/year for the electronic version, and \$/year (+\$, if shipping outside the US) for print and electronic. Subscriptions, requests for back issues from the last three years and changes of subscribers address should be sent to MSP.

Analysis \& PDE (ISSN 1948-206X electronic, 2157-5045 printed) at Mathematical Sciences Publishers, 798 Evans Hall \#3840, c/o University of California, Berkeley, CA 94720-3840, is published continuously online. Periodical rate postage paid at Berkeley, CA 94704, and additional mailing offices.

APDE peer review and production are managed by EditFlow ${ }^{\circledR}$ from MSP.

PUBLISHED BY

- mathematical sciences publishers

nonprofit scientific publishing

http://msp.org/

(C) 2016 Mathematical Sciences Publishers 


\section{ANALYSIS \& PDE}

\section{Volume $9 \quad$ No. $3 \quad 2016$}

Local analytic regularity in the linearized Calderón problem

JOHANNES SJÖSTRAND and GUNTHER UHLMANN

Dispersive estimates for the Schrödinger operator on step-2 stratified Lie groups

Hajer Bahouri, Clotilde Fermanian-KAmmerer and Is ABelle Gallagher

Obstructions to the existence of limiting Carleman weights

Pablo Angulo-Ardoy, Daniel Faraco, Luis GuiJarro and Alberto Ruiz

Finite chains inside thin subsets of $\mathbb{R}^{d}$

Michael Bennett, Alexander Iosevich and Krystal Taylor

Advection-diffusion equations with density constraints

ALPÁR RICHÁRD MÉSZÁROS and FILIPPO SANTAMBROGIO

Asymptotic stability in energy space for dark solitons of the Landau-Lifshitz equation

YAKINE BAHRI

On the well-posedness of the generalized Korteweg-de Vries equation in scale-critical $\hat{L}^{r}$ space

SATOShi Masaki and JUN-ICHI SEgatA

Regularity for parabolic integro-differential equations with very irregular kernels

RUSSELL W. SCHWAB and LuIS SilvestRe 